Review

\title{
Segmentation Methods of Echocardiography Images for Left Ventricle Boundary Detection
}

\author{
${ }^{1}$ Fatima Khalid and ${ }^{1}$ Rohollah Moosavi Tayebi \\ ${ }^{I}$ Faculty of Computer Science and Information Technology, UPM, Malaysia \\ ${ }^{2}$ Cardiothoracic Unit, Surgical Cluster, Faculty of Medicine, UiTM, Malaysia
}

${ }^{1}$ Samaneh Mazaheri, ${ }^{1}$ Rahmita Wirza, ${ }^{1}$ Puteri Suhaiza Sulaiman, ${ }^{2}$ Mohd Zamrin Dimon,

Article history

Received: 02-06-2015

Revised: 02-10-2015

Accepted: 05-11-2015

Corresponding Author:

Samaneh Mazaheri

Faculty of Computer Science

and Information Technology,

UPM, Malaysia

Email: mazaheri.samaneh@yahoo.com

\begin{abstract}
Due to acoustic interferences and artifacts which are inherent in echocardiography images, automatic segmentation of anatomical structures in cardiac ultrasound images is a real challenge. This paper surveys stateof-the-art researches on echocardiography data segmentation methods, concentrating on methods techniques developed for clinical data. We present a classification of methodologies for echocardiography image segmentation. By choosing ten recent papers which have proposed innovative ideas that they proved certain clinical advantages or potential especial role to the echocardiography segmentation task. The contribution of the paper would be serving as a tutorial of the field for both clinicians and technologists, providing large number of segmentation techniques in a comprehensive and systematic manner and critically review recent approaches in terms of their performance and degree of clinical evaluation with respect to the final goal of cardiac functional analysis.
\end{abstract}

Keywords: Segmentation, Echocardiography, Active Contours Methods, Level Set (LS) Approaches, Deformable Templates

\section{Introduction}

The echocardiography and cardiac Computed Tomography (CT) are emerging diagnostic tools among modern imaging modalities for visualizing cardiac structure and diagnosing cardiovascular diseases. The echocardiography (Anderson, 2007) is real-time, non-invasive imaging modality which is less expensive than $\mathrm{CT}$ and Magnetic Resonance imaging (MR). Recently, the problem of automatic detection, segmentation and tracking of heart chambers in radiological imaging, such as ultrasound and $\mathrm{CT}$, have received considerable attentions (Frangi et al., 2001). Quality of echocardiography images influence directly on segmentation result. There are some artifacts such as attenuation, speckle, shadows and signal dropout which make the segmentation process difficult; because of orientation dependence for acquisition data, result can lead to missing borders. Since the contrast between areas of interest is usually low, segmentation task in ultrasound images turns to a challenging one (Noble and Boukerroui, 2006). Although, recently the quality of information from an ultrasound device has substantially improved, due to recent advances in transducer design, spatial/temporal resolution, digital systems, portability, etc. (Bridal et al., 2003). Due to these advances use of echocardiography has been increased in many ways; not only the traditional scope of application, CAD and diagnosis, but also new areas like therapy and image guided interventions. Therefore, currently, there is an urge in understanding how to do image segmentation, one of the oldest image processing tasks, to echocardiography data.

In ultrasound data, the LV appearance is mainly characterized by a dark region, representing the blood pool inside the chamber, enclosed by the endocardium, myocardium and epicardium, which are roughly depicted by a brighter region (Fig. 1). In ultrasonic devices, there is a great variability of the gray value distribution and the spatial texture in each of the above-mentioned regions (Santiago et al., 2013). This happens among different ultrasound sequences and within the same sequence. This is due to the several reasons: Fast motion during systole phase, low signal-to-noise ratio, edge dropout (especially in the diastole phase) and the presence of shadows produced by the dense muscles, the specific properties and settings of the ultrasound machine and the anisotropy of the ultrasonic image formation (Bosch et al., 2002). 


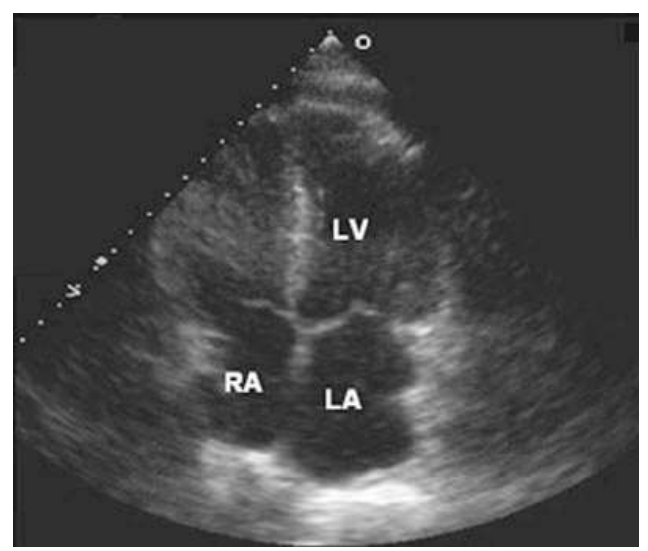

Fig 1. Echocardiography; apical four-chamber view (Chan and Veinot, 2011)

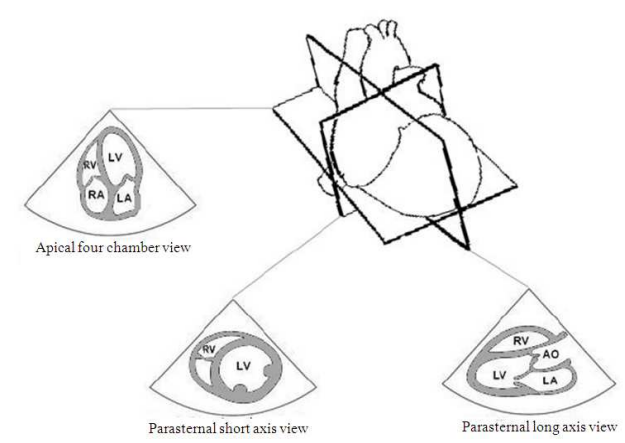

Fig 2. Explanation of different views in echocardiography (Lohr and Sivanandam, 2009)

Echocardiography images can be used for many different tests: Cardiac structure, heart development, function and also changes in normal physiologic states and pathologic conditions. For example, Left ventricular function can be obtained through 2 dimensional echocardiography images by calculating the ejection fraction. Depending on where on the chest the user positions the transducer, different views of the heart can be obtained. Some of the most common views are: Long axis view of the left atrium and the left ventricle, short axis views of the heart in planes from the base of the heart to the apex and the four chambers view; Fig. 2.

Previous works in our lab towards the goal of Cardiac Intervention Environment, Cardiovascular Information System, and Heart Diseases Diagnostic Systems has included an automatic coronary arterial tree extraction in angiograms (Moosavi Tayebi et al., 2014), cardiac ultrasound fusion system development (Mazaheri et al., 2014), Wavelet enhancement for x-ray angiography (Moosavi Tayebi et al., 2015), 3D Multimodal Cardiac Data Reconstruction in CT Angiography (Moosavi Tayebi et al., 2015), CT Angiography components categorization and coronary artery enhancement (Moosavi Tayebi et al., 2014), and some surveys including review on segmentation approaches (Mazaheri et al., 2013 and Moosavi Tayebi et al., 2014), and review on registration of cardiac images Mazaheri et al. (2015).
The paper is organized as follows: In section II, we review the ideas in the field of segmentation methods; section III presents an overview of the proposed segmentation approaches for echocardiography images; section IV reviews the latest developments; and finally, section V concludes the paper with final remarks.

\section{Segmentation Methods}

Most considerations have been given to tracking the motion of the endocardium, e.g., blood pool or tissue border to allow for approximation of left ventricular volumes or areas and derived measures such as the ejection fraction and for regional wall motion analyzing. Especially, these measures are used in assessment and diagnosis of ischaemic heart disease. Most analysis is based on $2 \mathrm{D}$ acquisitions in which it is implicitly assumed that the principal component of motion is in the plane of the acquisition slice. Parasternal Short-Axis (SAX) is the standard 2D diagnostic views used for this and apical two-chamber (2C), Four-Chamber (4C) and Three-Chamber (3C) views. The latter three are also sometimes referred to as (apical) Long-Axis (LAX) views. The quality of data and therefore issues for segmentation, vary regarding to the view due to the anisotropy of ultrasound image acquisition, artifacts like attenuation and shadowing from the lungs which can be severe. Segmentation methods should also have strategies for avoiding the papillary muscles. Reliably finding the epicardial border, i.e., outer wall is more challenging, especially from apical views.

Lots of the preliminary works concentrated on one frame segmentation yet; considering maximum expansion or end diastole and maximum contraction or end systole frames to calculate some measurements like EF (the ejection fraction). Although, analysis should be done for the whole cardiac cycle for fully assess heart function. Cardiologists also use a movie of a heart in decisionmaking as the speckle pattern associated with de-forming tissue can be observed in a movie whereas in a still frame the speckle pattern is not always useful. As a result and in contrast to some other clinical application areas, in echocardiography it is perhaps more logical to view segmentation as a spatio-temporal problem. Lots of recent successful techniques have taken this point of view. A priori research of endocardial boundary detection is proposed in (Hammoude, 1998). In Fig. 3, there are some examples of segmentation results from (Bridal et al., 2003).

Echocardiography images suffer from several specific drawbacks, which impede both human interpretation and automated analysis.

- There is no simple relation between pixel intensity and any physical property of the tissue visualized. Echocardiography images are formed as a combination of interference patterns (speckle) and reflections at tissue transitions. 
Different tissues are mostly not distinguishable by their intensity values or texture

- Echocardiographic image information is highly anisotropic and position dependent, since reflection intensity, spatial resolution and signal to noise ratio depend on the depth and the angle of incidence of the echocardiography beam, as well as of user controlled depth gain settings

- Many imaging artifacts occur, resulting in local loss of anatomical information: Significant amounts of noise, dropouts (for structures parallel to the echocardiography beam), shadowing (behind acoustically dense structures), side lobes, reverberations and limited echo windows. Stillframe images, therefore, often only contain partial information

For these reasons, automated segmentation of echocardiographic image sequences has proven to be a challenging task. Many approaches to segmentation echocardiographic data have been proposed. They can be divided into the following classes: Level Set (LS) approaches, Deformable templates, Active Shape Models (ASM), Active contours methods, Active Appearance Models (AAM), Bottom-up approaches and Databaseguided (DB-guided) segmentation (Santiago et al., 2013).

\section{Level Set Segmentation Approaches}

The use of level sets for medical image segmentation aims at improving the performance of active contours due to the following. First, LS are able to increase robustness of the model by combining both region and boundary segmentation. Second, the texture and shape priors are jointly used with a continuous parametric function to model the implicit segmentation function (Bernard et al., 2007; 2009; Cremers et al., 2006; Lin et al., 2003; Lynch et al., 2008; Paragios and Deriche,
2002; Sarti et al., 2005; Debreuve et al., 2001; Paragios, 2003).

\section{Deformable Templates}

Alternatively, these issues can also be faced using deformable templates (Chen et al., 2008; Duan et al., 2010; Nascimento and Marques, 2008; Zagrodsky et al., $2005)$ that use an unsupervised scheme for learning. However, deformable template-based methods require the knowledge of how the initialization is performed.

Both level-sets and deformable templates have demonstrated good results when dealing with medical images. Nonetheless, they also present some drawbacks regarding the prior knowledge included in the optimization function.

\section{Active Shape Models (ASM) and Active Appearance Models (AAM)}

The previously raised issues have also motivated the development of the supervised based models, in which the shape and appearance of the LV are learned from a manually annotated training set. This class of methods includes the Active Shape Model (ASM) (Cootes et al., 1995; Parker et al., 1994; Cootes et al., 1994) and Active Appearance Model (AAM) (Bosch et al., 2002; Cootes et al., 1999; Mitchell et al., 2001). However, both methods need a large set of annotated training images and the initialization must be close to a local optimum. Furthermore, these methods assume a Gaussian distribution of the shape and appearance derived from the training samples.

\section{Active Contours Segmentation Methods}

Active contours methods inspired the development of Level Set (LS) methods (Malladi et al., 1995; Kass et al., 1987; Chalana et al., 1996; Hozumi et al., 1997) which significantly reduce the sensitivity to initial conditions.

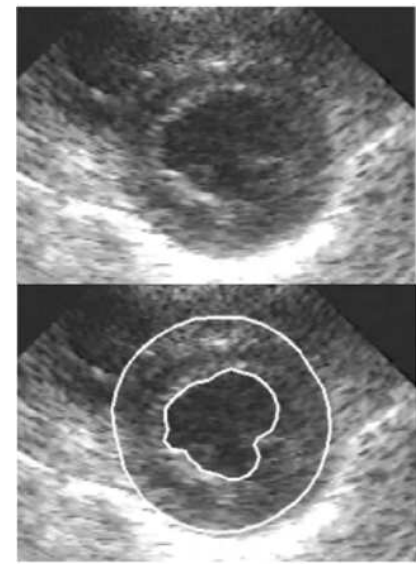

(a)

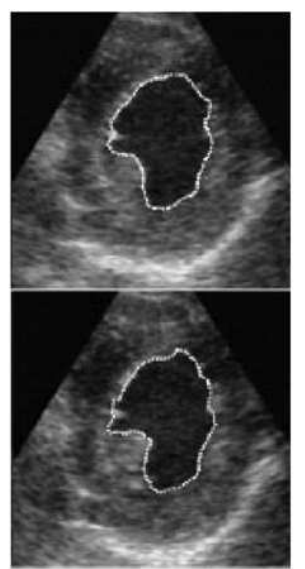

(b)

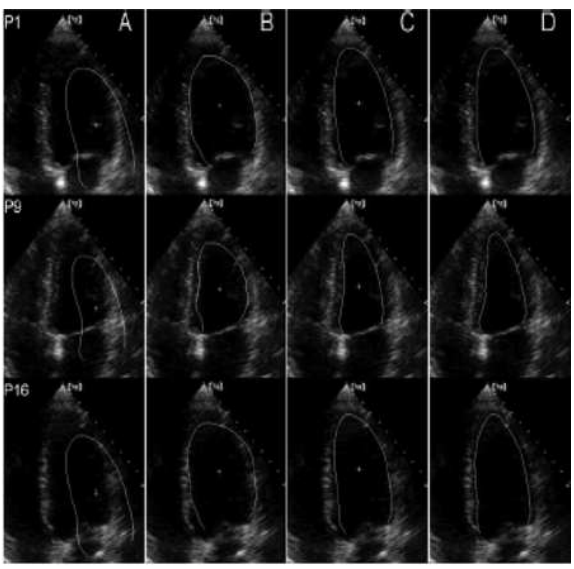

(c)

Fig 3. Some reults of echocardiography image segmentation. Short Axis images (SAX) from (a) (Dias and Leitão, 1996) and from (b) (Mikic et al., 1998) (c) Long Axis images (LAX) from (Bosch et al., 2002) 


\section{Bottom-up Segmentation Approaches}

Bottom-up approaches detect the LV boundary using edge detection that constitutes features to represent the object boundary. Although these methods have low computational complexity, they are sensitive to initial conditions and generally lack robustness to imaging conditions (Sonka et al., 1995; Zhang and Geiser, 1984).

\section{Database-Guided (DB-Guided) Segmentation}

The above issues motivated the proposal of the DB-guided segmentation approaches that use supervised learning techniques (Georgescu et al., 2005; Zhou et al., 2005). Specifically, discriminative learning model based on boosting techniques (Freund and Schapire, 1997) is developed to segment LV from ultrasound images. Another important point in the DBguided approach is its independence regarding an initial guess. Instead of that, a full search is conducted in the parameter space. However, these methods have several drawbacks. Besides the high complexity of the search process, super-vised learning methods face two main difficulties which are the large number of training images (in the order of hundreds) needed to estimate the parameters of the model and the robustness to imaging conditions absent from the training set (Carneiro and Nascimento, 2010; Carneiro et al., 2008; Zhou et al., 2005).

Though partially successful, three major problems are associated with many of the existing echocardiographic contour detection strategies.

- The current methods typically do not include information about the allowable range of shape and appearance variations of the segmented objects. Ultrasonic image information is often illdefined or incomplete. Therefore, extensive model knowledge about the characteristic organ shape and appearance, its anatomical and pathological shape variations and spatial organ embedding should form an integral part of a robust segmentation approach

- Most existing methods use implicit, global and oversimplified models for the contour location. The location of strong local image features, however, does not always correspond to the desired contour as drawn by an expert human observer. The exact location of the contour cannot always be determined from the strongest image evidence, but should be modeled or learned from examples provided by expert observers. Moreover, contour characteristics vary for different parts of the local (and still unknown) anatomy

- Many automated techniques perform a static segmentation on single $2 \mathrm{D}$ frames or use rudimentary continuity constraints and, therefore, often produce segmentation results that are inconsistent with the dynamics of the cardiac cycle. An expert observer, however, utilizes knowledge about cardiac contraction dynamics and temporal coherence of structures and texture to resolve ambiguities and to determine the exact LV boundary location, mostly after reviewing the image data in a cine loop. Recently, several methods have been reported that try to deal with the third problem in time sequences of either 2D or $3 \mathrm{D}$ echocardiograms

Nonetheless, 3D echocardiography has gained increasing interest and several methods to perform the $3 \mathrm{D}$ segmentation of the $\mathrm{LV}$ have become available in literature (Noble and Boukerroui, 2006). One approach to perform 3D segmentation is to consecutively applying 2D segmentations to each image plane and assembling them into a 3D structure (Nillesen et al., 2006; Scowen et al., 2000) as cardiologists manually do in such cases. However, such approaches require additional methods to pre-vent spatial inconsistencies in the surface. Other approaches have performed the 3D segmentation using 3D active contours such as level-set (Hang et al., 2004; Yu et al., 2006; Juang et al., 2011). Furthermore, over the last decade some effort has been put into developing $3 \mathrm{D}+\mathrm{t} \mathrm{LV}$ tracking systems that are able to segment the LV over the course of the cardiac cycle, such as (Orderud et al., 2007; Yang et al., 2008).

\section{Echocardiography Image Segmentation Approaches}

\section{2-Dimensional Endocardial Boundary Estimation}

For echocardiography endocardial segmentation, finding contour method is the most common technique which has been considered. Since contrast surrounding the boundary of the left ventricle differs relating to the appropriate situation of the transducer orientation and also artifacts and attenuation, the boundary would not be clear. Therefore, conventional gradient intensity based techniques don't have success on common ultra-sound data.

Mishra et al. (2003) presented an active contour approach which for optimization used a genetic algorithm. Two experts have done manual descriptions on twenty frames and the usual amount compared with the automatic technique to indicate that the inter-variability among experts was resemble to the difference between the manual and automatic techniques. Mignotte and Meunier (2001) for the segmentation of short axis parasternal images, apply a statistical external energy in a discrete active contour and discussed this is work best for echocardiography 
images which they have huge noise and disappeared borders. With this aim, a shifted Rayleigh distribution was applied to show the statistics of gray levels.

Mignotte et al. (2001) suggested a border detection algorithm, using Bayesian framework which used deformable templates for modeling the precedent information. This technique is unsupervised and fully automatic. The approximation problem which is formulated as a Maximum A Posteriori (MAP) optimization solved via a genetic algorithm. They also presented a comparison to manual descriptions which is done by two experts. Boukerroui et al. (2003) proposed the same Bayesian methodological method where the segmentation is region-based. They used level sets which they are usually considered as an alternative to active contours for echocardiography image segmentation.

Lin et al. (2002) proposed a different level set segmentation approach where fuses region and edge information with spatial scales. For this method they considered a closed curve as a border. They applied segmentation method to single 2D slices, while used their proposed method to rotational 3D echocardiography images. When the quality of echocardiography images is good, their method work well. Yan and Zhuang (2003) used an adaptation of the fast marching cube technique for applying the level set technique on echocardiography data. They used a mean gradient intensity based measure to deduct errors related to local feature measurements. They discussed the results only quantitatively, but applied their algorithm to an apical four chamber view and a SAX (parasternal short axis) view.

Boukerroui et al. (2003), proposed an accurate adaptive region segmentation which works in a Bayesian frame-work. The adaptive property for compensating existence inhomogeneity and non-uniformity of echocardiography data within the same tissue, considers a slow spatial variation and local class mean. They evaluated their method by manual description which is done by an observer on a LAX (long axis view) and compared with another segmentation which is also region based method, Xiao et al. (2002).

ANN-based (Artificial neural network) techniques also have been used for region based segmentation (Rekeczky et al., 1999; Noble and Boukerroui, 2006). For example, Binder et al. (1999) applied a 2 layer back propagation network to end-systolic and end-diastolic SAX images (parasternal short axis view) from thirtyeight patients with different quality data which consist of 12 images with good quality, 13 images with moderate quality and 13 images with poor quality. This is one of the few researches that have done experience at data of different quality. Result of segmentation was successful in thirty-four of the thirty-eight database. The automatic technique was compared with manual tracking which is done by 2 experts.

\section{Spatio-Temporal Methods $(2 D+T)$}

We can consider echocardiography image segmentation as a Spatio-temporal problem, as the Region Of Interest (ROI) changes in a non-rigid manner and it has inhomogeneity over the frames. Spatio-temporal examination gives a local approximate of image velocity along with segmentation and leads to more suitable localization of a boundary. For example, Mulet-Parada and Noble (2000) presented a local-phase-based approach for detection endocardial border with Spatio-temporal space. They showed that local phase would be a better basic for echocardiography feature detection and also segmentation; because local phase is fixed to intensity magnitude. They used a derived measure from phase, which is called feature asymmetry for identifying the endocardium in a $2 \mathrm{D}+\mathrm{T}$ space (Spatio-temporal). Since then local phase approach has been used for feature identify in lots of ultrasound researches (Mulet-Parada, 2000; Ye et al., 2002; Sanchez-Ortiz et al., 2002).

Mikic and others presented an active contour technique, provided by the method of Singh and Allen (1992; Boukerroui et al., 2003), which propagation of a contour from one single frame to another one was guided by optical flow approximates (Mikic et al., 1998). This information can be used for determining the initialization of an active contour. Metrics in active contour empirically determined. The approach was evaluated on 3 long axis, 3 short axis and 2 aortic roots images; and compared with three to five manual delineations (Fig. 3a).

Dias and Leitão (1996), presented an approach for the both epicardial and endocardial boundary approximation like the former research of Friedland and Adam (1989) and also Teles de Figueiredo and Leitaa (1992). In (Friedland and Adam, 1989; Teles de Figueiredo and Leitaa, 1992), they used polar coordinates for contour description; although, as far as we know, this is the first method which considers noise statistics in border optimization. They modeled the estimation problem in a Bayesian frame-work as a MAP estimation problem with a Spatio-temporal MRF for the regularization term and Rayleigh statistics for the data term. For solving the optimization problem, they used a repetitive multi-grid dynamic programming algorithm. They presented results on one short axis view image and also on synthetic data. Figure 3(a) shows an example result from (Teles de Figueiredo and Leitaa, 1992).

Figueiredo et al. (2000), inside the same framework, proposed a parametric contour segmentation in an unsupervised way, based on B-spline descriptions. They applied a MDL (minimum description length) parameter to estimate B-spline knots' number.

Jacob et al. (1999; 2001; 2002) proposed a Kalman filter based epicardial and endocardial boundary 
tracing technique based on contour tracking method of (Blake and Isard, 1998). They built a Spatio-temporal contour model where it has 2 sections; a shape model made from manual description of the left ventricle boundaries of a motion model and a training set.

Bosch et al. (2002) presented the Active Appearance Motion Model (AAMM) to represent the appearance and shape of the endocardium, also its motion by applying an adaption of the Active Appearance Model (AAM) technique; Fig. (3c) shows an example of this technique. Mitchell et al. (2002) proposed a 3D active appearance model for segmentation of 4 chamber cardiac ultrasound images (2D with the third dimension time, 2D+T). This technique adjusts itself in time and space; it means it is Spatio-temporal. They trained the model from manually segmented instances in a learning phase.

\section{Endocardial Boundary Detection in $3 D$ and $4 D$ Images}

In previous research on 3D endocardial boundary detection, Coppini et al. (1995) have taken into account reconstruction and segmentation of Left Ventricle (LV) from freehand echocardiography images which they are captured at 4 set angles regarding to the 4 chambers view.

Song and others considered the segmentation process in a Bayesian framework as a surface fitting problem which finding a $3 \mathrm{D}$ surface where has the largest previous probability is the aim (Song et al., 2002). Wolf and others (Wolf, et al., 2002) proposed the Restricted Optimal Path Exploring Segmentation (ROPES) method, where is a semi-automatic segmentation. They applied their method to 2D slices of 3D TEE (transesophageal echocardiography) data.

Angelini et al. (2001) considered segmentation of real time 3D echocardiographic images from a volumetric process. Montagnat et al. (2003) extended a $3 \mathrm{D}+\mathrm{T}$ model based segmentation method for rotational 3D echocardiographic data. This research used a combined edge and region-based approach and for analysis it uses a cylindrical geometry. Especially, the orientation and intensity gradient magnitude are applied, together with a region-based on a local analysis of intensity values. The answers are set in a variational frame and some of their features make them well suited to 3D echocardiographic segmentation. Sarti et al. (1999) proposed a model for multiple scale analysis of space time echocardiography images which trying to solve a nonlinear (partial) differential equation fusing proposed methods of the Galilean invariant movie multi scale analysis of Guichard with anisotropic diffusion model from Perona Malik (Guichard, 1998; 1994). In (Mikula et al., 2000), authors extended spatial diffusion from Perona Malik and substituted it with a nonlinear anisotropic smoothing curvature driven level sets (Preußer and Rumpf, 2002). Then, Mikula and others proposed a truly-coupled Spatiotemporal anisotropic diffusion (Mikula et al., 2004). In this research, time smoothing was curvature driven and they discussed, it resulted in better smoothing with velocity discontinuity preservation in compare with the last acceleration-based smoothing.

Corsi et al. (2002) applied a level-set based segmentation algorithm to real-time 3D echocardiographic data from a volumetric system. The level-set's initialization is defined by manual delineation on a few Short Axis frames (SAX). Sarti and others proposed a level set segmentation method for images, like echocardiographic data, that can have missing borders (Sarti et al., 2000; 2002). This method differs completely with anterior level-set segmentation methods that consider just the zero level-set. Their research was later developed by (Corsaro et al., 2004) and Mikula et al., (2004); a robust and accurate semi-implicit numerical volume scheme was presented to solve the Riemannian mean curvature flow equation in 2D (Mikula et al., 2004) and 3D (Corsaro et al., 2004). Examples of segmentation from classical Kanizsa triangle were presented to show that the technique can find missing borders. They also presented some examples on 3D cardiac (Mikula et al., 2004) and fetal echocardiography images (Sarti et al., 2000).

\section{Myocardium and Epicardium Segmentation}

Eventually, for analysis and segmentation of myocardium, there is a very limited literature (Kerut et al., 2000) and also for epicardial boundary estimation (Dias and Leitão, 1996; Feng et al., 1991; Malassiotis and Strintzis, 1999). These two tasks are both difficult to do directly on B-mode images. Also it is done a little research for finding methods which work on general clinical data rather than data from subjects with a good acoustic window. We addressed to some of the few researches which have done so above (Bosch et al., 2002; Binder et al., 1999; Mitchell et al., 2002). One further exception is the work of Boukerroui et al. (2001) which considers how to enhance B-mode images to reduce the effect of attenuation and enhance features. Although that meth-od was shown quantitatively to reduce attenuation, enhance features and not introduce artifacts after enhancement, that approach has not to date been fully tested in clinical practice. 


\section{Review the Latest Developments}

Kucera and Martin (1997) presented an approach with an external force region based in their previous research for LV segmentation. In an active contour 3D model, they defined this force with time as one dimension. The proposed technique is relatively reliable on Short and Long Axis (SAX and LAX) views.

Sarti et al. (2005) applied a region based method in their proposed segmentation technique which they assemble a prior knowledge for statistical distribution of gray levels. They used a level set technique to drive the curve evolution to get a maximum likelihood segmentation of the target, regarding to the statistical distribution law of image pixels. When comparing the area enclosed by the resulting contours from this technique with manually outlined contours the correlation is excellent.

Sarti et al. (2005) also proposed a region-based segmentation in their adaptive segmentation approach. They use a weighting function that considers both global and local statistics during the segmentation process. The segmentation of the left ventricle shows good results when compared with manual outlines by a medical expert.

Mishra et al. (2003) use an active contour model when segmenting the left ventricle in Short Axis (SAX) view. They solve the optimization problem with using a Genetic Algorithm (GA) and the performance is com-parable with interobserver variations.

Mignotte and Meunier (2001) proposed a multiscale approach to the contour optimization. Their external energy in the snake energy function is also region based. They demonstrated some segmentation results for Short Axis (SAX) views that are qualitatively good.

Chen et al. (2007) built an active contour algorithm in a geometric way with a prior intensity and shape. The results of applying the method to a 2 chamber view are promising.

Bosch et al. (2002) proposed an Active Appearance Motion Model (AAMM) that was used to do a segmentation of the left ventricle. This is an extension of Active Appearance Models (AAM) and they did an automated segmentation over the full heart cycle. Their results are comparable with inter-observer variations.

Mitchell et al. (2002) did a fully 3 dimensional Active Appearance Models (AAM) with time as one of the dimensions in echocardiography images. They obtained a correlation coefficient of 0.79 when comparing the area of the Left Ventricle (LV) defined by their method and an observer respectively.
Several other methods have also been evaluated for this segmentation problem, including a fuzzy multiscale edge detector (Setarehdan and Soraghan, 1999), artificial neural Networks (Binder et al., 1999; Rekeczky et al., 1999) and a Kalman filter based tracking method (Jacob et al., 2002).

All of these methods give acceptable segmentations of the Left Ventricle (LV) in Long Axis (LAX) and/or Short Axis (SAX) views.

Santiago et al. (2013) offered a bottom-up deformable-based model for the segmentation of the Left Ventricle (LV) in 3D ultrasound data. The presented methodology is based on Probabilistic Data Association Filter (PDAF). After a rough initialization given by the user, the following steps are performed: (1) low-level transition edge points are detected based on a prior model for the intensity of the LV, (2) middle-level features or patch formation is accomplished by linking the low-level information, (3) data interpretations are computed based on the reliability (belonging or not to the LV boundary) of the previously obtained patches, (4) a confidence degree is assigned to each data interpretation and the model is updated taking into account all data interpretations.

Landgren et al. (2013) presented a semi-automated segmentation method that used a region based snake. To avoid any unwanted concavities in the segmentations due to the cardiac valve they use two anchor points in the snake that are located to the left and to the right of the cardiac valve respectively. For the possibility of segmentations in different stages of the heart cycle these anchor points are tracked through the cycle. This tracking is based both on the resemblance of a region around the anchor points and a prior model of the movement in the ydirection of the anchor points. The tracking of the anchor points also seems to work well. The use of a prior model turned out to be necessary when testing the tracking algorithm without it. With no prior model the anchor points have a tendency to wander away or not follow the cardiac valve back when the heart relaxes.

McManigle et al. (2012) proposed a two-step Hough transform and introduced a myocardial segmentation technique based on the circular Hough transform. They developed a preliminary segmentation technique which takes advantage of both endocardial and epicardial image information. The segmentations generated are com-pared to expert manual segmentation of $3 \mathrm{D}+\mathrm{t}$ echocardiogram data and evaluated for their appropriateness to initialize a subsequent segmentation step.

Skalski et al. (2012) presented an application of active contour without edges method to left ventricle 
segmentation in ultrasound echocardiographic images. The proposed procedure consists of three basic modules: ROI calculation by means of Hough transform, image denoising by means of SRAD filtration and, finally, image segmentation by means of active contour without edges method. The proposed scheme can be modified and in conjugation with deformable image registration methods, e.g., B-Spline Free Form Deformation or Spring Mass system can be used for calculation of deformations of hearts structures like heart walls.

Pearlman et al. (2012) presented an algorithm for segmenting left ventricular endocardial boundaries from RF ultrasound. Their method incorporates a computationally efficient linear predictor that exploits short-term spatio-temporal coherence in the RF data. Segmentation is achieved jointly using an independent identically distributed spatial model for RF intensity and a multi-frame conditional model that relates neighboring frames in the image sequence. Segmentation using the RF data overcomes challenges due to image inhomogeneities often amplified in Bmode segmentation and provides geometric constraints for RF phase-based speckle tracking. The incorporation of multiple frames in the conditional model significantly increases the robustness and accuracy of the algorithm.

Dietenbeck et al. (2012) they proposed a method to segment the whole myocardium (endocardial and epicardial con-tours) in 2D echographic images. This is achieved using a level set model constrained by a new shape formulation that allows modeling both contours. Also their framework allows segmenting the whole myocardium for the four main views used in clinical routine. They approximated the heart boundaries by two hyper-quadrics that are then used as a shape prior for the evolving contour. The method is validated on a dataset of clinical images and compared with expert segmentation.

Robust and fast 3D tracking of deformable objects, such as heart, is a challenging task because of the relatively low image contrast and speed requirement. Many existing 2D algorithms might not be directly applied on the 3D tracking problem. The 3D tracking performance is limited due to dramatically increased data size, landmarks ambiguity, signal drop-out or complex non-rigid deformation. Yang et al. (2011) presented a 3D tracking algorithm, Prediction based Collaborative Trackers (PCT) and tested in both 3D ultrasound and CT. Compared with tracking by detection and 3D optical flow, PCT provides the best results. They demonstrated that PCT increases the tracking accuracy and especially speed dramatically. Their tracker is tested on three clinical datasets for three 3D heart tracking problems with two different imaging modalities: endocardium tracking of the left ventricle, dense tracking in the myocardial regions between the epicardium and endocardium of the left ventricle and whole heart four chambers tracking.

Recently, introduced variant of the level set method called level set without edges. This variant takes ad-vantage of the intensity value of area information instead of module of gradient which is typically used. Such approach guarantees stability and correctness of algorithm working on the border between object and back-ground with small absolute value of image gradient.

Skalski and Turcza (2011) proposed an algorithm for heart shape estimation (segmentation) in ECHO images using this level set method without edge. They proceeded the image segmentation with automatic Region Of Interest (ROI) calculation. The main idea of ROI calculations is to receive a triangle-like part of the acquired ECHO image, using linear Hough transform, thresholding and simple mathematics. The described algorithm involves the following three steps: Removing multiplicative noise from an image by nonlinear diffusion filtration, determination of diagnostically relevant area by Hough image transformation and finally image segmentation with active contour algorithm. Erroneous situations with pixels representing background are classified as an object by an algorithm were excluded by the proper restriction of the segmented area and controlling contour evolution process using information other than image gradient magnitude.

Juang et al. (2011) proposed a method to automatically segment the left ventricle and atrium without any user input in 3D ultrasound volumes. Their method can segment volumes with open and closed mitral valves. They utilized the radial symmetry transform to determine a central axis along which the $3 \mathrm{D}$ volume is warped into a cylindrical coordinate space. A graph is constructed for the volume in this space and a min-cut algorithm is applied to segment the left ventricle and atrium from the background. The segmented results are transformed back to the Cartesian coordinate space. This method can be extended to other types of segmentation techniques, such as level sets and watershed. The method is generic enough to be applied to other imaging modalities such as MRI or CT (Table 1). 
Table 1. Validation of echocardiography image segmentation

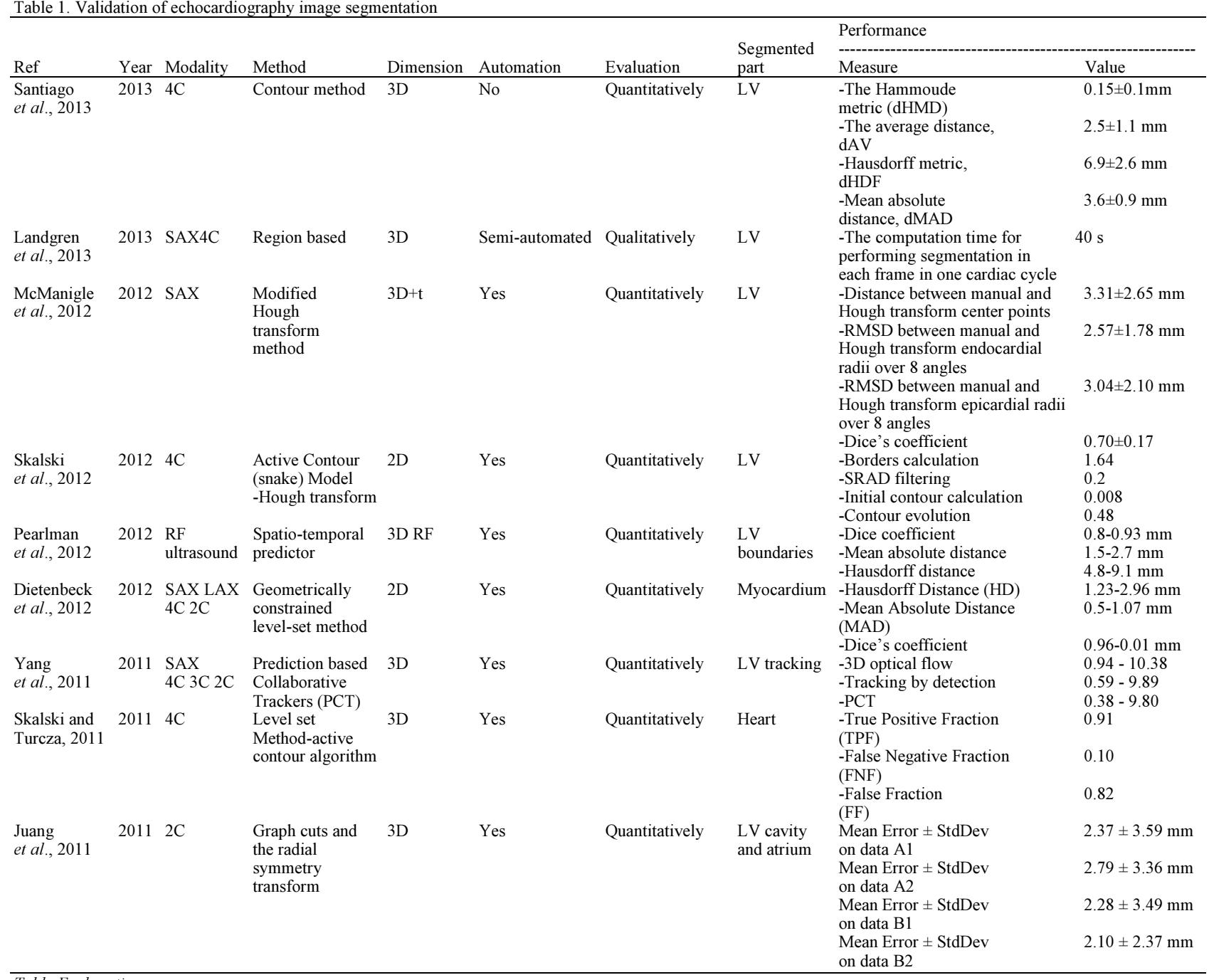

Table Explanations

Tables I summarize the evaluation which has been done on well-known techniques which explained in last Sections related to 6 common views echocardiography image for segmentation.

Key to table:

- $\quad$ Modality: $\mathrm{SAX}=$ Short Axis, $\mathrm{LAX}=$ Long Axis, $\mathrm{XC}=$ apical $\mathrm{X}$ Chamber $(\mathrm{X}=2,3,4)$

- Performance Metric: as mentioned, since researches are different in their measurements and naming, it is so difficult to set them through the studies

- Automation: (yes) full, (no) interactive guidance/correction

\section{Conclusion}

Usually, for examining medical image segmentation techniques in a quantitative way, we can use animal mod-el studies, phantom studies, simulation and manual delineation; one modality as a "reference" or "gold standard", or some relation between clinical result. Mostly, clinical info is utilized in evaluation. However, there is some literature on echocardiography segmentation techniques shows that some phantom and simulation studies also have been done. This somehow indicates problems in defining real phantoms and simulations. The most popular metric of performance evaluation is manual delineation on clinical data; although regarding to clinical area, there can be huge inter-expert and intraexpert variability; it is due to the fact that manual segmentation is not a routine task that they normally do. Therefore, delineation can be difficult to do. So, when manual delineation is applied as the gold standard, we should to take care in explaining results. Because there is no standardization of performance metrics, it is hard to compare techniques directly. That's why, in some researches, they use multiple experts to define the reference. We have made comparison between methods which proposed by research groups, since there is no standard database to compare results. Lacking of a standard dataset is confusing, since quality of echocardiography images varies a lot, in compare with other clinical imaging such as CT or MRI. 


\section{Funding Information}

This research was supported by Ministry of Science, Technology and Innovation, Malaysia, under Science Fund number UPM0007353.

\section{Authors' Contributions}

All authors have made substantial contributions to conception and design, data acquisition, analysis and interpretation of the data. All authors read and approved the final manuscript.

\section{Ethics}

The authors declare that there are no conflicts of interest.

\section{References}

Anderson, B., 2007. Echocardiography: The Normal Examination and Echocardiographic Measurements. 2nd Edn., MGA Graphics, Australia, ISBN-10: 0646468634, pp: 337.

Angelini, E.D., A.F. Laine, S. Takuma, J.W. Holmes and S. Homma, 2001. LV volume quantification via spatiotemporal analysis of real-time 3-D echocardiography. IEEE Trans. Med. Imag., 20: 457-469. DOI: $10.1109 / 42.929612$

Bernard, O., B. Touil, A. Gelas, R. Prost and D. Friboulet, 2007. A rbf-based multiphase level set method for segmentation in echocardiography using the statistics of the radiofrequency signal. Proceedings of the IEEE International Conference on Image Processing, Sept. 16-Oct. 19, IEEE Xplore Press, San Antonio, pp: 157-160. DOI: $10.1109 /$ ICIP.2007.4379270

Bernard, O., D. Friboulet, P. Thevenaz and M. Unser, 2009. Variational b-spline level-set: A linear filtering approach for fast deformable model evolution. IEEE Trans. Imag. Processing, 18: 1179-1991. DOI: 10.1109/TIP.2009.2017343

Binder, T., M. Sussner, D. Moertl, H. Strohmer and T. Baumgartner et al., 1999. Artificial neural networks and spatial temporal contour linking for automated endocardial contour detection on echocardiograms: A novel approach to determine left ventricular contractile function. Ultrasound Med. Biol., 25: 1069-1076. DOI: 10.1016/S0301-5629(99)00059-9

Blake, A. and M. Isard, 1998. Active Contours. 1st Edn., Springer-Verlag London, New York, ISBN-10: 9781-4471-1555-7, pp: 352.3

Bosch, J.G., S.C. Mitchell, B.P.F. Lelieveldt, F. Nijland and O. Kamp et al., 2002. Automatic segmentation of echocardiographic sequences by active appearance motion models. IEEE Trans. Med. Imag., 21: 1374-1383. DOI: 10.1109/TMI.2002.806427
Boukerroui, D., A. Baskurt, J.A. Noble and O. Basset, 2003. Segmentation of ultrasound imagesmultiresolution 2D and 3D algorithm based on global and local statistics. Pattern Recognit. Lett. 24: 779-790. DOI: 10.1016/S0167-8655(02)00181-2

Boukerroui, D., J.A. Noble and M. Brady, 2003. Velocity estimation in ultrasound images: A block matching approach. Proceedigs of the 18th International Conference on Information Processing in Medical Imaging, Jul. 20-25, Springer Berlin Heidelberg, UK, pp: 586-598. DOI: 10.1007/978-3-540-45087-0_49

Boukerroui, D., J.A. Noble, M.C. Robini and M. Brady, 2001. Enhancement of contrast regions in suboptimal ultrasound images with application to echocardiography. Ultrasound Med. Biol., 27: 1583-1594. DOI: 10.1016/S0301-5629(01)00478-1

Bridal, S.L., J.M. Correas, A. Saied and P. Laugier, 2003. Milestones on the road to higher resolution, quantitative and functional ultrasonic Imag. Proc. IEEE, 91: 1543-1561.

DOI: $10.1109 /$ JPROC.2003.817879

Carneiro, G. and J.C. Nascimento, 2010. Multiple dynamic models for tracking the left ventricle of the heart from ultrasound data using particle filters and deep learning architectures. Proceedings of the IEEE Conference on Computer Vision and Pattern Recognition, Jun. 13-18, IEEE Xplore Press, San Francisco, pp: 2815-2822.

DOI: $10.1109 /$ CVPR.2010.5540013

Carneiro, G., B. Georgescu, S. Good and D. Comaniciu, 2008. Detection and measurement of fetal anatomies from ultrasound images using a constrained probabilistic boosting tree. IEEE Trans. Med. Imag., 27: 1342-1355. DOI: 10.1109/TMI.2008.928917

Chalana,V., D.T. Linker, D.R. Haynor and Y. Kim, 1996. A multiple active contour model for cardiac boundary detection on echocardiographic sequences. IEEE Trans. Med. Imag., 15: 290-298.

DOI: $10.1109 / 42.500138$

Chan, K.1. and J.P. Veinot, 2011. Anatomic Basis of Echocardiographic Diagnosis. 1st Edn., Springer Science and Business Media, London, ISBN-10: 1849963878, pp: 491.

Chen, T., J. Babb, P. Kellman, L. Axel and D. Kim, 2008. Semiautomated segmentation of myocardial contours for fast strain analysis in cine displacement-encoded MRI. IEEE Trans. Med. Imag., 27: 1084-1094. DOI: 10.1109/TMI.2008.918327

Chen, Y., F. Huang, H. Tagare and M. Rao, 2007. A coupled minimization problem for medical image segmentation with priors. Int. J. Comput. Vis., 71: 259-272. DOI: 10.1007/s11263-006-8524-2

Cootes, T.F., A. Hill, C.J. Taylor and J. Haslam, 1994. Use of active shape models for locating structures in medical images. Image Vision Comput., 12: 355-366. DOI: 10.1016/0262-8856(94)90060-4 
Cootes, T.F., C. Beeston, G.J. Edwards and C.J. Taylor, 1999. A unified framework for atlas matching using active appearance models. Proceedings of the Information Processing in Medical Imaging, Jun. 28-Jul. 2, Springer Berlin Heidelberg, Hungary, pp: 322-333. DOI: 10.1007/3-540-48714-X_24

Cootes, T.F., C.J. Taylor, D.H. Cooper and J. Graham, 1995. Active shape models-their training and application. Comput. Vision Image Understand., 61: 38-59. DOI: $10.1006 /$ cviu. 1995.1004

Coppini, G., R. Poli and G. Valli, 1995. Recovery of the 3-D shape of the left ventricle from echocardiographic images. IEEE Trans. Med. Imag., 14: 301-317. 10.1109/42.387712

Corsaro, S., K. Mikula, A. Sarti and F. Sgallari, 2004. Semi-implicit covolume method in 3D image segmentation. Tech. Rep., Dept. Math., Slovak Univ. Technol., Bratislava, Slovak Republic.

Corsi, C., G. Saracino, A. Sarti and C. Lamberti, 2002. Left ventricular volume estimation for real-time three-dimensional echocardiography. IEEE Trans. Med. Imag., 21: 1202-1208.

DOI: $10.1109 /$ TMI.2002.804418

Cremers, D., S.J. Osher and S. Soatto, 2006. Kernel density estimation and intrinsic alignment for shape priors in level set segmentation. Int. J. Comput. Vision, 69: DOI: 10.1007/s1 1263-006-7533-5

Debreuve, E., M. Barlaud, G. Aubert, I. Laurette and J. Darcourt, 2001. Space-time segmentation using level set active contours applied to myocardial gated SPECT. IEEE Trans. Med. Imag., 20: 643-659. DOI: $10.1109 / 42.932748$

Dias, J.M.B. and J.M.N. Leitão, 1996. Wall position and thickness estimation from sequences of echocardiographic images. IEEE Trans. Med. Imag., 15: 25-38. DOI: 10.1109/42.481438

Dietenbeck, T., M. Alessandrini, D. Barbosa, J. D'hooge and D. Friboulet et al., 2012. Detection of the whole myocardium in 2D-echocardiography for multiple orientations using a geometrically constrained levelset. Med. Image Anal., 16: 386-401.

DOI: 10.1016/j.media.2011.10.003

Duan, Q., E.D. Angelini and A.F. Laine, 2010. Real time segmentation by active geometric functions. Comput. Meth. Prog. Biomed., 98: 223-230. DOI: 10.1016/j.cmpb.2009.09.001

Feng, J., W.C. Lin and C.T. Chen, 1991. Epicardial boundary detection using fuzzy reasoning. IEEE Trans. Med. Imag., 10: 187-199.

DOI: $10.1109 / 42.79477$

Figueiredo, M.A., J.M.N. Leitão and A.K. Jain, 2000. Unsupervised contour representation and estimation using B-splines and a minimum description length criterion. IEEE Trans. Med. Imag., 9: 1075-1087. DOI: $10.1109 / 83.846249$
Frangi, A.F., W.J. Niessen and M.A. Viergever, 2001. Three-dimensional modeling for functional analysis of cardiac images: A review. IEEE Trans. Med. Imag., 20: 2-25. PMID: 11293688

Freund, Y. and R.E. Schapire, 1997. A decision-theoretic generalization of on-line learning and an application to boosting. J. Comput. Syst. Sci., 55: 119-139. DOI: $10.1006 /$ jcss. 1997.1504

Friedland, N. and D. Adam, 1989. Automatic ventricular cavity boundary detection from sequential ultrasound images using simulated annealing. IEEE Trans. Med. Imag., 8: 344-353. DOI: $10.1109 / 42.41487$

Georgescu, B., X.S. Zhou, D. Comaniciu and A. Gupta, 2005. Database-guided segmentation of anatomical structures with complex appearance. Proceedings of the IEEE Computer Society Conference on Computer Vision and Pattern Recognition, Jun. 2025, IEEE Xplore Press, pp: 429-436.

DOI: 10.1109/CVPR.2005.119

Guichard, F., 1994. Axiomatisation des analyses multiéchelles d'images et de films. Ph.D. Thises, Univ. Paris IX Dauphine, Paris, France.

Guichard, F., 1998. A morphological, affine and galilean invariant scale-space for movies. IEEE Trans. Imag. Process., 7: 444-456. DOI: 10.1109/83.661194

Hammoude, A., 1998. Endocardial border identification in two-dimensional echocardiographic images: Review of methods. Comput. Med. Imag. Graph., 22: 181-193. DOI: 10.1016/S0895-6111(98)00024-X

Hang, X., N.L. Greenberg and J.D. Thomas, 2004. Left ventricle quantification in $3 \mathrm{D}$ echocardiography using a geometric deformable model. Proceedings of the Computers in Cardiology, Sept. 19-22, IEEE Xplore Press, Chicago, pp: 649-652. DOI: $10.1109 /$ CIC.2004.1443022

Hozumi, T., K. Yoshida, H. Yoshioka, T. Yagi and T. Akasaka et al., 1997. Echocardiographic estimation of left ventricular cavity area with a newly developed automated contour tracking method. J. Amer. Society Echocardiogr., 10:. 822-829. DOI: 10.1016/S0894-7317(97)70042-7

Jacob, G., J.A. Noble, A.D. Kelion and A.P. Banning, 2001. Quantitative regional analysis of myocardial wall motion. Ultrasound Med. Biol., 27: 773-784. DOI: 10.1016/S0301-5629(01)00350-7

Jacob, G., J.A. Noble, C. Behrenbruch, A.D. Kelion and A.P. Banning, 2002. A shape-space-based approach to tracking myocardial borders and quantifying regional left-ventricular function applied in echocardiography. IEEE Trans. Med. Imag., 21: 226-238. DOI: 10.1109/42.996341

Jacob, G., J.A. Noble, M. Mulet-Parada and A. Blake, 1999. Evaluating a robust contour tracker on echocardiographic sequences. Med. Image Anal., 3: 63-75. DOI: 10.1016/S1361-8415(99)80017-6 
Juang, R., E.R. McVeigh, B. Hoffmann, D. Yuh and P. Burlina, 2011. Automatic segmentation of the leftventricular cavity and atrium in 3D ultrasound using graph cuts and the radial symmetry transform. Proceedings of the IEEE International Symposium on Biomedical Imaging: From Nano to Macro, Mar. 30-Apr. 2, IEEE Xplore Press, Chicago, pp: 606609. DOI: $10.1109 /$ ISBI.2011.5872480

Kass, M., A. Witkin and D. Terzopoulos, 1987. Snakes: Active contour models. Int. J. Comput. Vision, 4: 321-331. DOI: 10.1007/BF00133570

Kerut, E.K., M.B. Given, E. McIlwain, G. Allen and C. Espinoza et al., 2000. Echocardiographic texture analysis using the wavelet transform: Differentiation of early heart muscle disease. Ultrasound Med. Biol., 26: 1445-1453.

DOI: $10.1016 / \mathrm{S} 0301-5629(00) 00289-1$

Kucera, D. and R.W. Martin, 1997. Segmentation of sequences of echocardiographic images using a simplified 3D active contour model with regionbased external forces. Comput. Med. Imag. Graph., 21: 1-21. DOI: 10.1016/S0895-6111(96)00027-4

Landgren, M., N.C. Overgaard and A. Heyden, 2013. Segmentation of the left heart ventricle in ultrasound images using a region based snake. In: Medical Imaging 2013: Image Processing, Ourselin, S., D.R. Haynor (Eds.), SPIE, USA, ISBN-10: 9780819494436, pp: 1-9.

Lin, N., W. Yu and J.S. Duncan, 2002. Combinative multi-scale level set framework for echocardiographic image segmentation, Proceedings of the 5th International Conference Medical Image Computing and Computer-Assisted Intervention, Sept. 25-28, Springer Berlin Heidelberg, Tokyo, pp: 682-689. DOI: $10.1007 / 3-540-45786-084$

Lin, N., W. Yu and J.S. Duncan, 2003. Combinative multi-scale level set framework for echocardiographic image segmentation. Med. Imag. Anal. 7: 529-537. DOI: $10.1016 /$ S1361-8415(03)00035-5

Lohr, J.L. and S. Sivanandam, 2009. Introduction to Echocardiography. In: Handbook of Cardiac Anatomy, Physiology and Devices, Iaizzo, P.A. (Ed.), Springer Science and Business Media, New York, ISBN-10: 1603273719, pp: 319-330.

Lynch, M., O. Ghita and P.F. Whelan, 2008. Segmentation of the left ventricle of the heart in 3$\mathrm{D}+\mathrm{t}$ MRI data using an optimized nonrigid temporal model. IEEE Trans. Med. Imag., 27: 195-203. DOI: 10.1109/TMI.2007.904681

Malassiotis, S. and M.G. Strintzis, 1999. Tracking the left ventricle in echocardiographic images by learning heart dynamics. IEEE Trans. Med. Imag., 18: 282-290. DOI: 10.1109/42.764905

Malladi, R., J.A. Sethian and B.C. Vemuri, 1995. Shape modeling with front propagation: A level set approach. IEEE Trans. Pattern Anal. Mach. Intell., 17: 158-175. DOI: $10.1109 / 34.368173$
Mazaheri, S., P.S. Sulaiman, R. Wirza, M.Z. Dimon and F. Khalid et al., 2015. Hybrid pixel-based method for cardiac ultrasound fusion based on integration of PCA and DWT. Computational Mathematical Methods Medicine, 2015: 1-16.

DOI: $10.1155 / 2015 / 486532$.

Mazaheri, S., P.S.B. Sulaiman, R. Wirza, F. Khalid and M.Z. Dimon et al., 2013. Echocardiography image segmentation: A survey. Proceeding of the 2nd International Conference on Advanced Computer Science Applications and Technologies, Dec. 23-24, IEEE Xplore, Kuching, pp: 327-332.

DOI: 10.1109/ACSAT.2013

Mazaheri, S., P.S.B. Sulaiman, R. Wirza, M.Z. Dimon and and F. Khalid et al., 2014. A Review of ultrasound and computed tomography registration approaches, Proceedings of the International Conference on Computer Assisted System in Health, Dec. 19-21, IEEE Xplore, Kuala Lumpur, pp: 6-11. DOI: $10.1109 /$ CASH.2014.17

McManigle, J.E., R.V. Stebbing and J.A. Noble, 2012. Modified hough transform for left ventricle myocardium segmentation in 3-D Echocardiogram images. Proceedings of the 9th IEEE International Symposium on Biomedical Imaging, May 2-5, IEEE Xplore Press, Barcelona, pp: 290-293.

DOI: 10.1109/ISBI.2012.6235541

Mignotte, M. and J. Meunier, 2001. A multiscale optimization approach for the dynamic contourbased boundary detection issue. Comput. Med. Imag. Graph., 25: 265-275. DOI: $10.1016 / \mathrm{S} 0895-6111(00) 00075-6$

Mignotte, M., J. Meunier and J.C. Tardif, 2001. Endocardial boundary E timation and tracking in echocardiographic images using deformable template and markov random fields. Pattern Anal. Appli., 4: 256-271. DOI: 10.1007/PL00010988

Mikic, I., S. Krucinski and J.D. Thomas, 1998. Segmentation and tracking in echocardiographic sequences: Active contours guided by optical flow estimates. IEEE Trans. Med. Imag., 17: 274-284. DOI: $10.1109 / 42.700739$

Mikula, K., A. Sarti and F. Sgallari, 2004. Handbook of Medical Image Analysis: Segmentation and Registration Models, Suri, J. (Ed.), Marcel Dekker Inc., New York.

Mikula, K., T. Preußer, M. Rumpf and F. Sgallari, 2000. On anisotropic geometric diffusion in $3 \mathrm{D}$ image processing and image sequence analysis. In: Trends in Nonlinear Analysis, Kirkilionis, M., S. Krömker, R. Rannacher and F. Tomi (Eds.), Springer Berlin Heidelberg, ISBN-10: 978-3-662-05281-5, pp: 307-321.

Mikula, K., T. Preußer and M. Rumpf, 2004. Morphological image sequence processing. Comput. Vis. Sci., 6: 197-209. DOI: $10.1007 / \mathrm{s} 00791-004-0129-0$ 
Mishra, A., P.K. Dutta and M.K. Ghosh, 2003. A GA based approach for boundary detection of left ventricle with echocardiographic image sequences. Image Vis. Comput., 21: 967-976. DOI: $10.1016 / \mathrm{S} 0262-8856(03) 00121-5$

Mitchell, S.C., B.P.F. Lelieveldt, R.J. van der Geest, H.G. Bosch and J.H.C. Reiver et al., 2001. Multistage hybrid active appearance model matching: Segmentation of left and right ventricles in cardiac MR images. IEEE Trans. Med. Imag., 20: 415-423. DOI: 10.1109/42.925294

Mitchell, S.C., J.G. Bosch, B.P.F. Lelieveldt, R.J. van der Geest and J.H.C. Reiber et al., 2002. 3-D active appearance models: Segmentation of cardiac MR and ultrasound images. IEEE Trans. Med. Imag., 21: 1167-1178. DOI: 10.1109/TMI.2002.804425

Moosavi Tayebi, R., P.S.B. Sulaiman, R. Wirza, M.Z. Dimon and S. Kadiman et al., 2014. A fast and accurate method for automatic coronary arterial tree extraction in angiograms. J. Comp. Sci., 10: 2060-2076. DOI: $10.3844 /$ jcssp.2014.2060.2076

Moosavi Tayebi, R., R. Wirza, P. Suhaiza Binti Sulaiman, M. Zamrin Dimon and F. Khalid et al., 2015. Using wavelet for X-ray angiography enhancement. Agricultural Ecological and Medical Sciences.

Moosavi Tayebi, R., R. Wirza, P.S.B. Sulaiman, M.Z. Dimon and F. Khalid et al., 2015. 3D multimodal cardiac data reconstruction using angiography and computerized tomographic angiography registration. J. Cardiothoracic Surgery, 10: 1-25. DOI: $10.1186 / \mathrm{s} 13019-015-0249-2$

Moosavi Tayebi, R., R. Wirza, P.S.B. Sulaiman, M.Z. Dimon and F. Khalid et al., 2014. Cardiac components categorization and coronary artery enhancement in CT angiography, Proceedings of the International Conference on Computer Assisted System in Health, Dec. 19-21, IEEE Xplore, Kuala Lumpur, pp: 18-21. DOI: 10.1109/CASH.2014.19

Moosavi Tayebi, R., P.S.B. Sulaiman, R. Wirza, M.Z. Dimon and S. Kadiman et al., 2013. Coronary artery segmentation in angiograms with pattern recognition techniques-A survey. Proceedings of the International Conference on Advanced Computer Science Applications and Technologies, Dec. 23-24, IEEE Xplore, Kuching, pp: 321-326. DOI: $10.1109 /$ ACSAT.2013.70

Montagnat, J., M. Sermesant, H. Delingette, G. Malandain and N. Ayache, 2003. Anisotropic filtering for model-based segmentation of 4D cylindrical echocardiographic images. Pattern Recognit. Lett., 24: 815-828

DOI: $10.1016 / \mathrm{S} 0167-8655(02) 00184-8$

Mulet-Parada, M. and J.A. Noble, 2000. 2D+T acoustic boundary detection in echocardiography. Med. Image Anal. 4: 21-30.

DOI: $10.1016 / \mathrm{S} 1361-8415(00) 00006-2$
Mulet-Parada, M., 2000. Intensity independent feature extraction and tracking in echocardiographic sequences. Ph.D. Thesis, Dept. Eng. Sci., Univ. Oxford, Oxford, U.K.

Nascimento, J.C. and J.S. Marques, 2008. Robust shape tracking with multiple models in ultrasound images. IEEE Trans. Imag. Processing, 17: 392-406. DOI: $10.1109 /$ TIP.2007.915552

Nillesen, M.M., R.G.P. Lopata, I.H. Gerrits, L. Kapusta and H.J. Huisman et al., 2006. P3A-5 3D segmentation of the heart muscle in real-time $3 \mathrm{D}$ echocardiographic sequences using image statistics. Proceedings of the IEEE Ultrasonics Symposium, Oct. 2-6, IEEE Xplore Press, Vancouver, pp: 1987-1990. DOI: 10.1109/ULTSYM.2006.508

Noble, J.A. and D. Boukerroui, 2006. Ultrasound image segmentation: A survey. IEEE Trans Med. Imag., 25: 987-1010. DOI: 10.1109/TMI.2006.877092

Orderud, F., J. Hansgard and S.I. Rabben, 2007. Realtime tracking of the left ventricle in 3D echocardiography using a state estimation approach. Proceedings of the 10th International Conference on Medical Image Computing and Computer-Assisted Intervention, Oct. 29-Nov. 2, Springer Berlin Heidelberg, Australia, pp: 858-865. DOI: $10.1007 / 978-3-540-75757-3 \quad 104$

Paragios, N. and R. Deriche, 2002. Geodesic active regions and level set methods for supervised texture segmentation. Int. J. Comput. Vision, 46: 223-247. DOI: $10.1023 / \mathrm{A}: 1014080923068$

Paragios, N., 2003. A level set approach for shape-driven segmentation and tracking of the left ventricle. IEEE Trans. Med. Imag., 22: 773-776. DOI: $10.1109 /$ TMI.2003.814785

Parker, A.D., A. Hill, C.J. Taylor, T.F. Cootes and X.Y. Jin et al., 1994. Application of point distribution models to the automated analysis of echocardiograms. Proceedings of the Computers in Cardiology, Sep. 25-28, IEEE Xplore Press, Bethesda, pp: 25-28. DOI: 10.1109/CIC.1994.470258

Pearlman, P.C., H.D. Tagare, B.A. Lin, A.J. Sinusas and J.S. Duncan, 2012. Segmentation of 3D radio frequency echocardiography using a spatio-temporal predictor. Med. Image Anal., 16: 351-360. DOI: 10.1016/j.media.2011.09.002

Preußer, T. and M. Rumpf, 2002. A level set method for anisotropic geometric diffusion in 3D image processing. SIAM J. Appl. Math., 62: 1772-1793. DOI: $10.1137 / \mathrm{S} 0036139901384662$

Rekeczky, C., A. Tahy, Z. Vegh and T. Roska, 1999. CNN-based spatio-temporal nonlinear filtering and endocardial boundary detection in echocardiography. Int. J. Circuit Theory Appli., 27:171-207.

Sanchez-Ortiz, G.I., G.J.T. Wright, J. Declerck, A. Banning and J. Noble, 2002. Automated 3-D echocardiography analysis compared with manual delineations and SPECT MUGA. IEEE Trans. Med. Imag., 21: 1069-1076. DOI: 10.1109/TMI.2002.804434 
Santiago, C., J.S. Marques and J.C. Nascimento, 2013. A robust deformable model for 3D segmentation of the left ventricle from ultrasound data. In: Mathematical Methodologies in Pattern Recognition and Machine Learning: Contributions from the International Conference on Pattern Recognition Applications and Methods, Carmona, L., P. Sánchez, J.S. Fred and A. Luisa (Eds.), Springer-Verlag New York, ISBN-10: 9781461450764, pp: 163-178.

Sarti, A., C. Corsi, E. Mazzini and C. Lamberti, 2005. Maximum likelihood segmentation of ultrasound images with rayleigh distribution. IEEE Trans. Ultrason. Ferroelectr. Frequency Control, 52: 947960. DOI: 10.1109/TUFFC.2005.1504017

Sarti, A., K. Mikula and F. Sgallari, 1999. Nonlinear multiscale analysis of three-dimensional echocardiographic sequences. IEEE Trans. Med. Imag., 18: 453-466. DOI: 10.1109/42.781012

Sarti, A., R. Malladi and J.A. Sethian, 2000. Subjective surfaces: A method for completing missing boundaries. Nat. Acad. Sci. USA, 12: 6258-6263. DOI: $10.1073 /$ pnas. 110135797

Sarti, A., R. Malladi and J.A. Sethian, 2002. Subjective surfaces: A geometric model for boundary completion. Int. J. Comput. Vis., 46: 201-221. DOI: $10.1023 / \mathrm{A}: 1014028906229$

Scowen, B., S. Smith and M. Vannan, 2000. Quantitative $3 \mathrm{D}$ modelling of the left ventrical from ultrasound images [ventrical read ventricle]. Proceedings of the Euromicro Conference, Sept. 5-7, IEEE Xplore Press, Maastricht, pp: 432-439. DOI: 10.1109/EURMIC.2000.874525

Setarehdan, S.K. and J.J. Soraghan, 1999. Automatic cardiac LV boundary detection and tracking using hybrid fuzzy temporal and fuzzy multiscale edge detection. IEEE Trans. Biomed. Eng., 46: 1364-1378. DOI: $10.1109 / 10.797997$

Singh, A. and P. Allen, 1992. Image-flow computation: An estimation-theoretic framework and a unified perspective. CVGIP: Image Understand., 56: 152-177. DOI: 10.1016/1049-9660(92)90037-4

Skalski, A. and P. Turcza, 2011. Heart segmentation in echo images. Metrol. Meas. Syst., 18: 305-314. DOI: $10.2478 / \mathrm{v} 10178-011-0012-\mathrm{y}$

Skalski, A., P. Turczaa, T. Zieliński, J. Królczykc and T. Grodzicki, 2012. Left ventricle usg image segmentation using active contour model. Procedia Comput. Sci., 1: 2723-2732.

DOI: 10.1016/j.procs.2010.04.306

Song, M.Z., R.M. Haralick, F.H. Sheehan and R.K. Johnson, 2002. Integrated surface model optimization for freehand three-dimensional echocardiography. IEEE Trans. Med. Imag., 21: 1077-1090. DOI: 10.1109/TMI.2002.804433

Sonka, M., X. Zhang, M. Siebes, M.S. Bissing and S.C. Dejong et al., 1995. Segmentation of intravascular ultrasound images: A knowledge-based approach. IEEE Trans. Med. Imag., 14: 719-732.

DOI: $10.1109 / 42.476113$
Teles de Figueiredo, M. and J.M.N. Leitaa, 1992. Bayesian estimation of ventricular contours in angiographic images. IEEE Trans. Med. Imag., 11: 416-429. DOI: $10.1109 / 42.158946$

Wolf, I., M. Hastenteufel, R. De Simone, M. Vetter and G. Glombitza et al., 2002. ROPES: A semiautomated segmentation method for accelerated analysis of three-dimensional echocardiographic data. IEEE Trans. Med. Imag., 21: 1091-1104. DOI: 10.1109/TMI.2002.804432

Xiao, G., M. Brady, J.A. Noble and Y. Zhang, 2002. Segmentation of ultrasound b-mode images with intensity inhomogeneity correction. IEEE Trans. Med. Imag., 21: 48-57. DOI: 10.1109/42.981233

Yan, J. and T. Zhuang, 2003. Applying improved fast marching method to endocardial boundary detection in echocardiographic images. Pattern Recognit. Lett. 24: 2777-2784. DOI: 10.1016/S0167-8655(03)00121-1

Yang, L., B. Georgescu, Y. Zheng, P. Meer and D. Comaniciu, 2008. 3D ultrasound tracking of the left ventricle using one-step forward prediction and data fusion of collaborative trackers. Proceedings of the IEEE Conference on Computer Vision and Pattern Recognition, Jun. 23-28, IEEE Xplore Press, Anchorage, pp: 1-8. DOI: $10.1109 /$ CVPR.2008.4587518

Yang, L., B. Georgescu, Y. Zheng, Y. Wang and P. Meer et al., 2011. Prediction Based Collaborative Trackers (PCT): A robust and accurate approach toward 3d medical object tracking. IEEE Trans. Med. Imag., 30: 1921-1932. DOI: 10.1109/TMI.2011.2158440

Ye, X., J.A. Noble and D. Atkinson, 2002. 3-D freehand echocardiography for automatic left ventricle reconstruction and analysis based on multiple acoustic windows. IEEE Trans. Med. Imag., 21: 1051-1058. DOI: 10.1109/TMI.2002.804436

Yu, H., M.S. Pattichis and M.B. Goens, 2006. Robust segmentation and volumetric registration in a multiview $3 \mathrm{~d}$ freehand ultrasound reconstruction system. Proceedings of the 40th Asilomar Conference on Signals, Systems and Computers, Oct. 29-Nov. 1, IEEE Xplore Press, Pacific Grove,pp: 1978-1982. DOI: $10.1109 /$ ACSSC.2006.355111

Zagrodsky, V., V. Walimbe, C.R. Castro-Pareja, J.X. Qin and J.M. Song et al., 2005. Registration-assisted segmentation of real-time 3-D echocardiographic data using deformable models. IEEE Trans. Med. Imag., 24: 1089-1099.

DOI: 10.1109/TMI.2005.852057

Zhang, L.F. and E.A. Geiser, 1984. An effective algorithm for extracting serial endocardial borders from 2-Dimensional Echocardiograms. IEEE Trans. Biomed. Eng., BME-31: 441-447. DOI: 10.1109/TBME.1984.325359

Zhou, X.S., D. Comaniciu and A. Gupta, 2005. An information fusion framework for robust shape tracking. IEEE Trans. Pattern Anal. Mach. Intell., 27: 115-129. DOI: 10.1109/TPAMI.2005.3 
Samaneh Mazaheri et al. / Journal of Computer Sciences 2015, 11 (9): 957.970 DOI: $10.3844 /$ jcssp.2015.957.970 